\title{
Demonstration of a Planar W-band, kW-level Extended Interaction Oscillator Based on a Pseudospark-sourced Sheet Electron Beam
}

\author{
G. X. Shu, H. Yin, L. Zhang, J. P. Zhao, G. Liu, A. D. R. Phelps, A. W. Cross, and W. He
}

\begin{abstract}
A W-band extended interaction oscillator (EIO) driven by a pseudospark-sourced sheet electron beam (PS-SEB) is demonstrated in this letter. The idea of combining the advantages of a pseudospark-sourced electron beam (high beam current density), a sheet electron beam geometry (large beam cross-sectional area) and a ladder-like slow wave structure (high gain per unit length) to generate powerful millimeter-wave radiation was experimentally verified. The PS-SEB based EIO produced $\sim 1.2 \mathrm{~kW}$ peak output power, an increase of six times in the measured power from an EIO based on a pseudospark-sourced pencil electron beam. Such a methodology offers a promising solution for portable, low-cost and powerful millimeter-wave and terahertz-wave radiation sources.
\end{abstract}

Index Terms - pseudospark-sourced sheet electron beam, extended interaction oscillator, high power millimeter-wave radiation source.

\section{INTRODUCTION}

$\mathrm{H}$ IGH power millimeter-wave vacuum electron devices (VEDs) have received considerable interest because of their diverse applications such as satellite communications, high resolution radar, and plasma science [1-3]. Compared with the electron beam generated by a conventional thermionic cathode electron gun, the pseudospark-sourced electron beam (PS-EB) has many advantages, including much higher beam current density $\left(>10^{8} \mathrm{~A} / \mathrm{m}^{2}\right)$, beam propagation without the need of an external guiding magnetic field, room-temperature operation, instant start-up and low vacuum requirement (50-500 mTorr) [4-11]. In addition, the PS-EB based VEDs can be fabricated using available technology such as wire erosion, which ensures the costs of production are moderate while at the same time a hand-held, portable, light-weight device can be manufactured. Several kinds of VEDs that used pencil-shaped PS-EB have been experimentally studied, including a Ka-band Cherenkov maser [5], a $0.2 \mathrm{THz}$ backward wave oscillator [6] and a W-band extended interaction oscillator (EIO) [8-9]. An EIO is one of the very promising powerful millimeter-wave and terahertz-wave radiation sources due to its advantages such as intensive beam wave interaction and short interaction length. The PS-EB driven EIO has been extensively studied at the University of Strathclyde in recent years and considerable efforts have been made to improve its performance [8-11]. A

G. X. Shu is with College of Electronic Science and Technology, Shenzhen University, Shenzhen, 518060, China. (e-mail: gxshu@szu.edu.cn)

H. Yin, L. Zhang, J. P. Zhao, W. He, A. D. R. Phelps, A. W. Cross are with Department of Physics, University of Strathclyde, Glasgow, G4 0NG, UK, (a.d.r.phelps@strath.ac.uk; a.w.cross@strath.ac.uk; w.he@strath.ac.uk)

G. Liu is with School of Physical Electronics, University of Electronic Science and Technology of China, Chengdu 610054, China. pencil PS-EB driven EIO was experimentally studied and a radiation pulse with $\sim 38 \mathrm{~W}$ peak power at $\sim 92 \mathrm{GHz}$ was measured [8]. The measured radiation power has been increased to $\sim 200 \mathrm{~W}$ after a post-acceleration of the pencil PS-EB was introduced in the pseudospark discharge system [9]. To further enhance the radiation power, this letter proposes to study the EIO using a pseudospark-sourced sheet electron beam (PS-SEB), exploiting the high beam current density of a PS-EB and the large cross sectional area of the sheet electron beam [12]. Furthermore the increased beam current was used to drive a resonant interaction circuit with a high characteristic impedance $(R / Q)$ to generate high radiation power. To verify the idea, a planar W-band EIO driven by a post-accelerated PS-SEB was designed, fabricated and experimentally studied.

\section{DESIGN AND ANALYSIS}

This novel EIO is mainly composed of a modified pseudospark discharge system being able to generate the PS-SEB and a planar interaction circuit. The pseudospark discharge system was modified from a system designed and experimentally studied in our early work [13]. The design and analysis of a $0.2 \mathrm{THz}$ similar planar interaction circuit has been presented in detail in our preliminary studies [10-11]. Differing from the EIO structure with a cylindrical beam tunnel [8-9], the planar interaction circuit has a rectangular beam tunnel. As shown in the inset of Fig. 1, the TM $\mathrm{TM}_{11}$-like mode excited in the ladder-like slow wave structure (SWS) cell has a longitudinal component of the electric field, ensuring the longitudinal beam wave energy exchange. As shown in Fig. 1, the $27.5 \mathrm{kV}$ beam line interacts with the dispersion curve of the operating mode near the phase difference of $2 \pi$, and the coupling impedance will rapidly increase when it approaches to $2 \pi$, resulting in a strong beam-wave interaction. As shown in Fig. 2 (b), the interaction circuit is composed of 13 SWS cells and an output coupling structure. Good performance was obtained for the optimized resonant SWS, including high $R / Q$ ( $R$ is the shunt impedance and $Q$ is the intrinsic quality factor) of 800 for the operating mode $\left(\mathrm{TM}_{110}-2 \pi\right.$ mode), which is much higher than the pencil PS-EB based EIO structure with $R / Q$ of 41.6 used in reference [8], contributing to stronger beam-wave interaction. A compact configuration for the optimized interaction circuit was obtained with 3-D dimensions of $6.6 \mathrm{~mm} \times 2.6 \mathrm{~mm} \times 13 \mathrm{~mm}$.

Simulation of beam-wave interaction was conducted to investigate the EIO performance using CST Particle Studio. A $29 \mathrm{kV}, 5 \mathrm{~A}$ sheet electron beam of $2.8 \mathrm{~mm} \times 0.5 \mathrm{~mm}$ in size was used to drive the EIO structure with a constant longitudinal magnetic field of $1 \mathrm{~T}$ used to approximate the focusing effect due to the ion channel $[6,8]$. To make the model closer to the 
actual situation, the energy spread of the electron beam and the conductivity of the background material was set as $20 \%$ and $\sigma_{\mathrm{Cu}} / 10\left(\sigma_{\mathrm{Cu}}=5.8 \times 10^{7} \mathrm{~S} / \mathrm{m}\right)$, respectively. A stable radiation signal with $5.1 \mathrm{~kW}$ power at $96.0 \mathrm{GHz}$ was observed. A radiation power of $1.5 \mathrm{~kW}$ was obtained when the beam current was $2 \mathrm{~A}$, considering a higher electron beam interception.

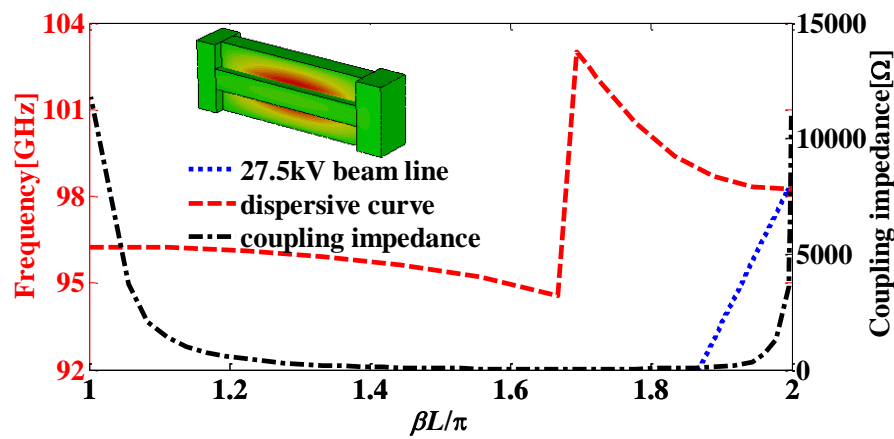

Fig.1. Dispersion curve and coupling impedance of the SWS cell and the 27.5 $\mathrm{kV}$ beam line. $\beta L$ is the phase difference, and $\beta$ and $L$ are the axial propagation constant and period of the SWS cell, respectively. Inset: $E_{\mathrm{z}}$-field distribution.

\section{FABRICATION AND EXPERIMENT}

As shown in Fig. 2 (a), a post-acceleration electrode was introduced between the cathode plate and the anode plate, and two perspex insulators were used for the insulation of the three brass plates. The interaction circuit was attached to the anode plate and a drift tube was placed at its end, which was connected with a rotary pump so as to keep the gas pressure in the whole system stable. To extract the sub-THz wave from the EIO, a transmitting horn was connected with the interaction circuit by a W-band rectangular waveguide WR-10. The interaction circuit was split into three parts in the fabrication. The beam tunnel and 13 gaps were wire cut from a copper block using a tungsten wire with diameter of $30 \mu \mathrm{m}$, which is shown in Fig. 2 (c). The reflection coefficient of the interaction circuit was measured using a vector network analyzer (Anritsu ME7808) and the trend of the curve was in good agreement with the simulated results, as shown in Fig. 3. There existed a frequency shift of $\sim 6 \mathrm{GHz}$ and the frequency interval of the operating $2 \pi$ mode $(104.6 \mathrm{GHz})$ and the nearest competitive mode $2 \pi / 12$ mode was measured to be $\sim 0.8 \mathrm{GHz}$.

When a high voltage pulse was applied across the system, the trigger gap between the cathode plate and the trigger electrode breaks down first and initializes the pseudospark discharge. After that, most of the discharge voltage will be applied over the pseudospark discharge gap between the cathode plate and the post-acceleration electrode, and the breakdown of the background gas with low pressure (0-10 $\mathrm{Pa}$ in this case) and the emission of the electrons will occur. At the final stage in a typical pseudospark discharge, a pencil-like PS-EB with high current density but low energy can be achieved at the end of the anode plate with a cylindrical aperture (3 $\mathrm{mm}$ diameter). If the beam energy can be increased to occur in the effective operating voltage range of the EIO, an efficient beam wave interaction can be achieved. To obtain such a PS-EB with combined high beam energy and beam current, the PS-EB generated at the final stage in a typical pseudospark discharge is post-accelerated by the voltage applied over the post-acceleration gap between the anode plate and the post-acceleration electrode. When the PS-EB propagates through a $2.8 \mathrm{~mm} \times 0.5 \mathrm{~mm}$ rectangular collimator placed within the anode, a PS-SEB can be obtained. The PS-SEB is focused by an ion channel formed by the ionization of the background gas and propagates forward for the beam wave interaction.

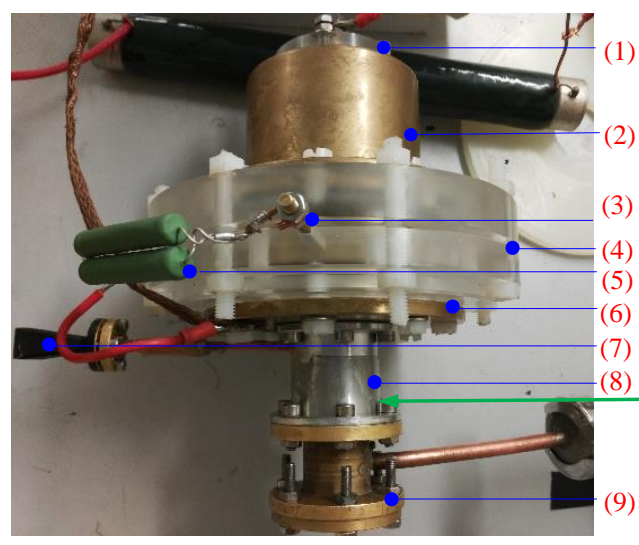

(a)

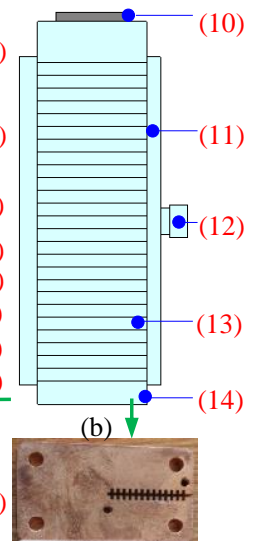

(c) (1)trigger, (2)cathode, (3)post-acc. electrode, (4)insulator,(5)post-acc. resistor, (6)anode, (7) transmitting horn, (8) interaction circuit, (9) drift tube (10) beam source, (11)coupling cavity, (12)output coupling structure, (13) gap, (14) beam tunnel.

Fig. 2. Photo of (a) the fabricated EIO, (b) the simulation model of the interaction circuit and (c) the fabricated part with the beam tunnel and 13 gaps.

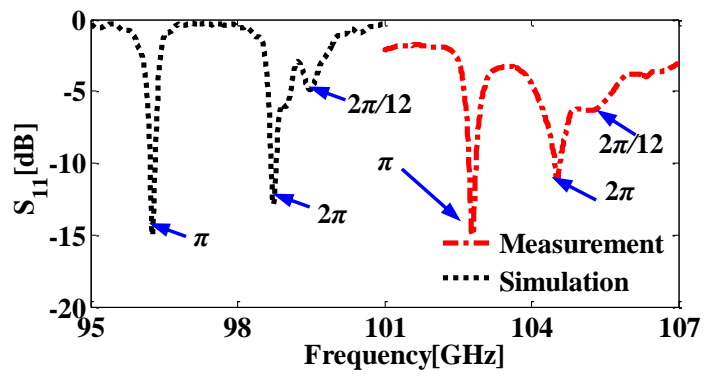

Fig. 3 Simulation and measurement results for the reflection curve $(\pi, 2 \pi / 12$, $2 \pi$ mode mean that the number of changes for the direction of the $E_{z}$-field along the 13 gaps is 12,2 , and 0 , respectively.)

To measure the beam current, the interaction circuit in Fig. 2 (a) was replaced by a Rogowski coil and the other components remained unchanged. The cathode voltage and the post-acceleration voltage were measured using two identical high voltage probes and were recorded on a Tektronix TDS 640 oscilloscope. A receiving horn was placed adjacent to the transmitting horn with a separation distance of $3 \mathrm{~cm}$. The radiation pulse was detected using an adjustable attenuator (-35 $\mathrm{dB}$ in this case) and a W-band crystal detector both located in a shielded metal box. To calibrate the detector, the EIO in the experimental setup was replaced by a $1.5 \mathrm{~W}$ QuinStar W-band solid-state source with other components remained unchanged. The detected voltage curve for different input power and different distance between the pair of horns was recorded, which was used to estimate the radiation power. To estimate the frequency range, several cylindrical waveguides with a series of radii, i.e. different cut-off frequencies were used. If the detected signal appears when using a waveguide with a lower cut-off frequency, but disappears when using a waveguide with a higher cut-off frequency, it can be confirmed that the radiated frequency lies between these two cut-off frequencies. 
Fig. 4 presents the typical time-correlated traces of the discharge voltage, beam current, and the radiation pulse. The cathode voltage had a longer pulse duration than the post-acceleration voltage, indicating the breakdown of the pseudospark discharge gap happened prior to that of the post-acceleration gap, which meant that the PS-EB had been successfully post-accelerated. The duration for beam current of over $5 \mathrm{~A}$ and meanwhile beam energy of over $28 \mathrm{keV}$ was up to $20 \mathrm{~ns}$. The maximum voltage $U$ and maximum current $I$ were $\sim 32 \mathrm{kV}$ and $\sim 30 \mathrm{~A}$, respectively. Based on the recorded millimeter-wave signal in Fig. 4, the attenuation used and the calibrated data of the detector, a peak output power $P_{\text {out }}$ of 1.2 $\mathrm{kW}$ and a FWHM (full width half maximum) of $\sim 20 \mathrm{~ns}$ were estimated. The oscillation frequency $f$ was measured to be in the frequency range of 104-106 GHz.

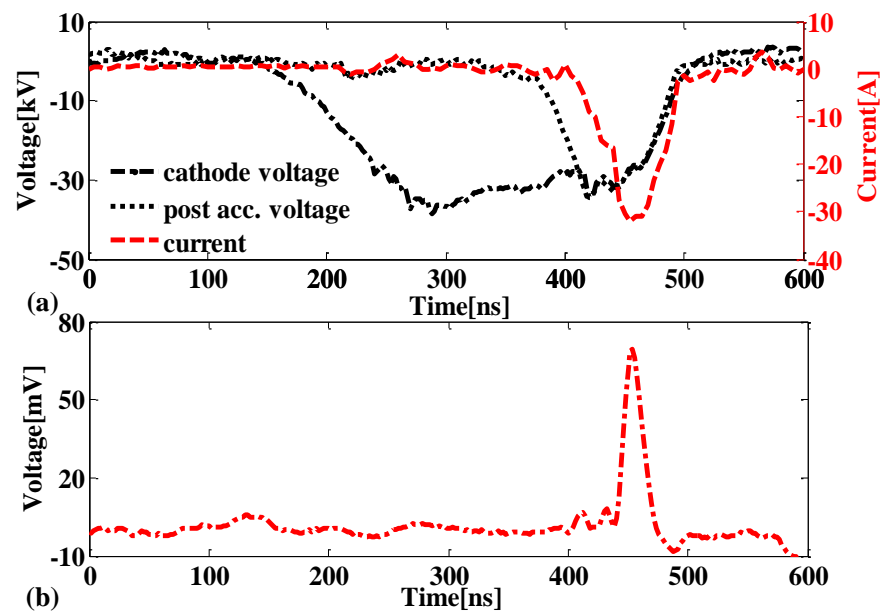

Fig. 4. (a) Measured time-correlated cathode voltage, post-acceleration voltage, beam current and (b) the detected voltage from the millimeter-wave detector.

Table 1 compares the experimental results of three W-band PS-EB based EIO experiments carried out at the University of Strathclyde. From table 1, the beam area of the PS-SEB (1.4 $\left.\mathrm{mm}^{2}\right)$ is seven times greater than that of the pencil PS-EB $(0.2$ $\mathrm{mm}^{2}$ ), indicating that the beam current taking part in the beam wave interaction will be much higher. Compared with the post-accelerated pencil PS-EB based EIO, the radiation power of the planar EIO was improved by a factor of 6 . In addition to the increased beam current, the much higher $R / Q$ of the planar SWS is another factor contributing to the higher power.

Table I Comparison of experimental results for three W-band PS-EB based EIOs constructed at the University of Strathclyde

\begin{tabular}{cccccccc}
\hline Ref. & $\begin{array}{c}\text { Beam } \\
\text { type }\end{array}$ & $\begin{array}{c}\text { Beam } \\
\text { size }[\mathrm{mm}]\end{array}$ & Post-acc. & $\begin{array}{c}U \\
{[\mathrm{kV}]}\end{array}$ & $\begin{array}{c}I \\
{[\mathrm{~A}]}\end{array}$ & $\begin{array}{c}P_{\text {out }} \\
{[\mathrm{kW}]}\end{array}$ & $\begin{array}{c}f \\
{[\mathrm{GHz}]}\end{array}$ \\
\hline $\begin{array}{r}\text { ref. } \\
{[8]}\end{array}$ & $\begin{array}{c}\text { Pencil } \\
\text { EB }\end{array}$ & $\begin{array}{c}0.5 \\
\text { diameter }\end{array}$ & No & $\sim 38$ & $<4$ & 0.038 & $>92$ \\
\hline $\begin{array}{r}\text { ref. } \\
{[9]}\end{array}$ & $\begin{array}{c}\text { Pencil } \\
\text { EB }\end{array}$ & $\begin{array}{c}0.5 \\
\text { diameter }\end{array}$ & Yes & 30 & 10 & 0.2 & $\begin{array}{c}92- \\
94\end{array}$ \\
\hline $\begin{array}{c}\text { this } \\
\text { letter }\end{array}$ & SEB & $2.8 \times 0.5$ & Yes & 32 & 30 & 1.2 & $\begin{array}{c}104- \\
106\end{array}$ \\
\hline
\end{tabular}

\section{CONCLUSION}

We have demonstrated the successful operation of a planar W-band EIO driven by a post-accelerated PS-SEB, which can produce $\sim 1.2 \mathrm{~kW}$ of peak output power. The radiation power of the PS-EB based EIO can be greatly improved after combining a sheet electron beam geometry with a ladder-like SWS. This methodology has provided a promising solution for reduction of costs and miniaturization in the realization of millimeter-wave radiation sources with high output power. It will be studied in the $\mathrm{THz}$ regime in our further work.

\section{ACKNOWLEDGMENT}

The authors would like to thank Mr David Barclay at the University of Strathclyde for machining the components of the EIO.

\section{REFERENCES}

[1] J. H. Booske, R. J. Dobbs, C. D. Joye, C. L. Kory, G. R. Neil, G. S. Park, J. Park, and R. J. Temkin, "Vacuum electronic high power terahertz sources," IEEE Trans. Terahertz Sci. Technol., vol. 1, no. 1, pp. 54-75, Sep. 2011, DOI: 10.1109/TTHZ.2011.2151610.

[2] G. Ulisse and V. Krozer, "W-band traveling wave tube amplifier based on planar slow wave structure," IEEE Trans. Electron Device Letters, vol. 38, no. 1, pp. 126-129, Jan. 2017, DOI: 10.1109/LED.2016.2627602.

[3] D. K. Abe, D. E. Pershing, K. T. Nguyen, F. N. Wood, R. E. Myers, E. L. Eisen, M. Cusick, and B. Levush, "Demonstration of an S-band, 600-kW fundamental-mode multiple-beam klystron," IEEE Trans. Electron Device Letters, vol. 26, no. 8, pp. 590-592, Aug. 2005, DOI: 10.1109/LED.2005.852540.

[4] N. Kumar, D. K. Pal, R. P. Lamba, U. N. Pal, and R. Prakash, "Analysis of geometrical design parameters for high-energy and high-current-density pseudospark-sourced electron beam emission," IEEE Trans. Electron Devices, vol. 64, no. 6, pp. 2688-2693, Jun. 2017, DOI: 10.1109/TED.2017.2696826.

[5] G. R. M. Robb, W. He, A. D. R. Phelps, A. W. Cross, and K. Ronald, "Pseudospark-based electron beam and Cherenkov maser experiments," Phys. Plasmas., vol. 7, no. 12, pp. 5195-5205, Dec. 2000, DOI: 10.1063/1.1319637.

[6] W. He, L. Zhang, D. Bowes, H. Yin, K. Ronald, A. D. R. Phelps, and A. W. Cross, "Generation of broadband terahertz radiation using a backward wave oscillator and pseudospark-sourced electron beam," Appl. Phys. Lett., 107, no. 13, pp. 133501-1-133501-4, Sep. 2015, DOI: 10.1063/1.4932099.

[7] N. Kumar, R. Lamba, A. Hossain, U. N. Pal, A. D. R. Phelps, and R. Prakash, "A tapered multi-gap multi- aperture pseudospark-sourced electron gun based X-band slow wave oscillator," Appl. Phys. Lett., 111, no. 21, pp. 213502-1-213502-4, Nov. 2017, DOI: 10.1063/1.5004227.

[8] Y. Yin, W. He, L. Zhang, H. Yin, C. R. Robertson, and A. W. Cross, "Simulation and experiments of a W-band extended interaction oscillator based on a pseudospark-sourced electron beam," IEEE Trans. Electron Devices, vol. 63, no. 1, pp. 512-516, Jan. 2015, DOI: 10.1109/TED.2015.2502950.

[9] J. Zhao, H. Yin, L. Zhang, W. He, Q. Zhang, A. D. R. Phelps, and A. W. Cross, "Experiments on W-band extended interaction oscillator with pseudospark sourced post-accelerated electron beam," Phys. Plasmas, vol. 24, no. 7, pp. 060703-1-060703-4, 2017, DOI: 10.1063/1.4985684.

[10]G. Shu, W. He, L. Zhang, H. Yin, J. Zhao, A. W. Cross, and A. D. R. Phelps, "Study of a $0.2-\mathrm{THz}$ extended interaction oscillator driven by a pseudospark-sourced sheet electron beam," IEEE Trans. Electron Devices, vol. 63, no. 12, pp. 4955-4960, Dec. 2016, DOI: 10.1109/TED.2016.2615314.

[11] G. Shu, L. Zhang, H. Yin, J. Zhao, A. D. R. Phelps, A. W. Cross, G. Liu, Y. Luo, Z. F. Qian, and W. He, "Experimental demonstration of a terahertz extended interaction oscillator driven by a pseudospark-sourced sheet electron beam," Appl. Phys. Lett., vol. 112, no. 4, Jan. 2018 (in press).

[12] A. Baig, D. Gamzina, T. Kimura, J. Atkinson, C. Domier, B. Popovic, L. Himes, R. Barchfeld, M. Field, and N. C. Luhmann, Jr. , "Performance of a nano-CNC machined $220-\mathrm{GHz}$ traveling wave tube amplifier," IEEE Trans. Electron Devices, vol. 64, no. 5, pp. 590-592, Aug. 2017, DOI: 10.1109/TED.2017.2682159.

[13] J. Zhao, H. Yin, L. Zhang, G. Shu, W. He, Q. Zhang, A. D. R. Phelps, and A. W. Cross, "Advanced post-acceleration methodology for pseudospark-sourced electron beam," Phys. Plasmas, vol. 24, no.2, pp. 023105-1-023105-4, Feb. 2017, DOI: 10.1063/1.4975188. 